\title{
New features and improvements in the NEA nuclear data tool suite
}

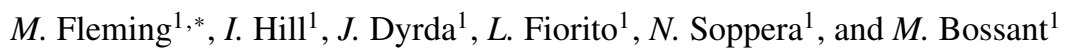 \\ ${ }^{1}$ OECD Nuclear Energy Agency, Boulogne-Billancourt, France
}

\begin{abstract}
The OECD Nuclear Energy Agency (NEA) has developed and maintains several products that are used in the verification and validation of nuclear data, including the Java-based Nuclear Data Information System (JANIS) and the Nuclear Data Sensitivity Tool (NDaST). These integrate other collections of the NEA, including the International Handbooks of benchmark experiments on Criticality Safety and Reactor Physics (ICSBEP and IRPhEP) and their supporting relational databases (DICE and IDAT). Recent development of the JANIS, DICE and NDaST systems have resulted in the ability to perform uncertainty propagation utilising Legendre polynomial sensitivities, calculation of case-to-case covariances and correlations, use of spectrum weighting in perturbations, calculation of statistical results with suites of randomly sampled nuclear data files and new command-line interfaces to automate analyses and generate XML outputs. All of the most recent, major nuclear data libraries have been fully processed and incorporated, along with new visualisation features for covariances and sensitivities, an expanded set of reaction channel definitions, and new EXFOR data types defined by the NRDC. Optimisation of numerical methods has also improved performance, with over order-ofmagnitude speed-up in the case of sensitivity-uncertainty calculations.
\end{abstract}

\section{Introduction}

The OECD Nuclear Energy Agency (NEA) Nuclear Science Committee (NSC) programme contains Working Parties in multiple disciplines, including International Nuclear Data Evaluation Co-operation (WPEC), Nuclear Criticality Safety (WPNCS) and Reactor Systems (WPRS). The latter two oversee Technical Review Groups for the International Criticality Safety Benchmark Evaluation Project (ICSBEP) and International Reactor Physics Evaluation (IRPhE) Project, which are internationally respected databases. To greatly increase the utility of these resources, as well as the nuclear data libraries shared within WPEC, the NEA established a Java application team to develop and maintain resources to explore and perform analyses on these databases. Over more than two decades, the NEA has created multiple professionallydeveloped Java tools of interest to the nuclear data community, including the JAva-based Nuclear Data Information System (JANIS) Database for the ICSBEP (DICE) and the Nuclear Data Sensitivity Tool (NDaST). Following brief descriptions for these tools in the following sections, we review the recent developments in each and discuss the future strategy for application development at the NEA.

\subsection{JANIS}

JANIS [1] is a visualisation and analysis program that allows users to interrogate evaluated and experimental nuclear data, compare different sources and perform various

*e-mail: michael.fleming@oecd-nea.org manipulations. Along with the software, the NEA has developed a database of nuclear data sources, including all of the major evaluated nuclear data libraries and experimental databases that the NEA Databank contributes to.

JANIS provides uniquely comprehensive visualisation and data-retrieval functionality for:

- processed and unprocessed ENDF-6 files;

- covariance data for cross sections, fission neutron spectra, fission neutron multiplicities and more (in various, but specific, formats);

- EXFOR [2] data, including preliminary Nuclear Reaction Data Centre (NRDC) transmissions;

- bibliographic experimental within the Computer Index of Nuclear Reaction Data (CINDA).

The JANIS database structure that can be integrated with other tools to utilise the central values and correlated uncertainties for analyses, for example using sensitivity profiles for some experimental system. The EXFOR handling of JANIS is so sophisticated in its error handling that the NRDC, which maintains and update the EXFOR database, requires JANIS checking for all preliminary submissions to the database.

Virtually all nominal MF and MT numbers in the ENDF6 format are handled by JANIS, including cross sections, decay data, fission product yields, emitted spectra, neutron multiplicities, prompt gamma spectra, isotope production and branching ratios, angular distributions, double differential data and more. 


\subsection{DICE}

The International Criticality Safety Evaluation Benchmark Project (ICSBEP) is a massive handbook containing thousands of evaluated experiments, each with detailed documents providing descriptions of the experiments, geometry and material compositions, uncertainty analyses and more. While it is a rich resource with many references, it is often impractical to trawl through ICSBEP looking for basic information, let alone identify similar experiments based on some quantifiable criteria. The NEA developed DICE to probe the handbook through only standard classification criteria, such as main fissile and spectra, as well any other standard information (e.g. years, laboratory, geometry, neutron balance, flux multi-group distributions, fissile ratios, reflector or fuel materials, and many more). These data were supplemented with reference calculations performed with Monte-Carlo transport codes using multiple libraries which are now included in the database.

Over the past years, as sensitivity calculation methodologies have become more sophisticated, thousands of sensitivity profiles to cross sections, nubar, fission neutron spectra and the first elastic Legendre polynomial coefficient have been calculated and added to the ICSBEP Handbook and DICE, as shown in Table 1. These can be used in new similarity search algorithms within DICE that allow users to identify similar benchmarks based on similar sensitivity profiles to specific reaction channels of identified isotopes. The inclusion of these sensitivity profiles within a relational database, as with the JANIS nuclear data, also offers the opportunity to use them for other analyses.

\subsection{NDaST}

Rather than add even more complex functionality to the feature-rich DICE, it was determined that a new application should be launched to utilise the JANIS nuclear data and DICE sensitivities. This is shown schematically in Figure 1, where perturbations may be calculated by calculating differences between two nuclear data quantities within JANIS and multiplying by the sensitivity within DICE, or correlated uncertainty matrices may be propagated using the first-order 'sandwich rule' $S C S^{T}$.

With the advent of near-complete sensitivity databases for all of ICSBEP, as shown in Table 1, and the already complete JANIS storage of evaluated nuclear data libraries, users can calculate uncertainties or the changes in some integral quantity due to a new evaluation on a set of dozens to thousands of cases within ICSBEP - all on the order of cpu-seconds to cpu-minutes. These can be broken down by component (e.g. perturbation of only the fission cross section) or by full files, as done during the CIELO project $[3,4]$ and shown in Figure 2. In the case shown, results for some 1250 benchmarks were calculated within cpu-seconds with trends shown for different aspects of the file. This demonstrates only the start of the analyses, as individual cases can be (and were) down-selected and probed by studying trends, the detailed sensitivity profiles and the differences in the evaluated files.

\section{Recent Developments in the NEA Nuclear Data Tool Suite}

The last JANIS-4.0 release was done in 2013, while a continuously-updated $\alpha$-version has been in development. The nuclear data libraries, EXFOR dictionaries and database have been routinely updated, with the most recent nuclear data libraries added shown in Table 2.

New covariance formats and search features have been added to JANIS, in part to accomodate the needs of NDaST to propagate these into benchmarks uncertainties. These include cross-channel and cross-MF correlations that can also be visualised, as shown in Figure 3.

A new command-line tool for interfacing with and extracting nuclear data quantities has been added due to user requests, and has been used to perform studies including Monte-Carlo elastic scattering sensitivity calculations to Legendre polynomial coefficients [5]. A new data import serialisation upgrade now uses SQL databases in memory to provide a greater than order-of-magnitude speed increase. These, along with various bugfixes and and GUI enhancements will be packaged into a late-2019 release of version 4.1.

As the most recent of the NEA nuclear data tools, NDaST has had a great deal of new developments since the ND2016 conference [6]. Recent features include the ability to calculate case-to-case correlations and representativity factors based on nuclear data similarity. Energy spectrum weighting has been added for perturbation calculations as well as the ability to handle the new elastic scattering 1st Legendre coefficient sensitivities that have been added to DICE. A new XML data input/output system has developed, allowing users to post-process their results with an intuitive schema for additional analyses outside the NDaST application. The ability to use perturbed or so-called 'random' files that reflect the uncertainty distributions of the underlying data has been added in a HalfMonte-Carlo uncertainty propagation system [7] that can use the TENDL files [8] or files generated with tools such as SANDY [9].

With comprehensive sensitivity profiles for all isotopes and reaction channels provided in DICE, new optimisations have included user-adjustable thresholds to significantly improve performance by isolating impactful sensitivities in mass calculations (while users may also probe the effects of impurities or minor channels, as required).

\section{Future Developments}

In keeping with the NEA mission, the Nuclear Science Division is continuing to develop the databases and software tools that integrate the outputs of different Working Parties, including those on nuclear data, criticality safety and reactor physics. Within WPEC, the Expert Group on the Recommended General Nuclear Data Structure (GNDS) is preparing the specifications for the nextgeneration, extensible nuclear data formats that will, in co-operation with Subgroup 48, include thermal scattering covariances. These groups work directly with NEA staff 
Table 1. History of the ICSBEP Handbooks and the inclusion of sensitivity profile data with data sources identified.

\begin{tabular}{lll}
\hline Handbook Edition & Number of Unique Cases & Sources \\
\hline 2012 & 727 & TSUNAMID+TSUNAMI3D[VALID]+MMK-KENO \\
2013 & 3575 & Previous + Non-VALID cases SCALE6.0 from Balance Inputs \\
2014 & 4011 & Previous + MCNP6 + SCALE6.2BClutch \\
2015 & 4065 & Previous + New Cases \\
2016 & $4200+$ & Previous + New Cases + 400 P1 Sensitivities \\
2017 & $4200+$ & Previous + 700 P1 Sensitivities \\
\hline
\end{tabular}

Nuclear data $(\sigma, v, \chi$, etc $)$

+ Covariances

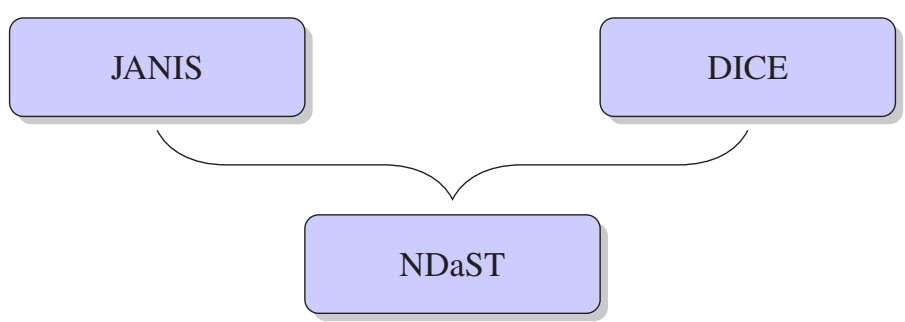

Perturbation calculations $(S P)$

and uncertainty propagation $\left(S C S^{T}\right)$

Figure 1. Simplified schematic explaining the database contents within JANIS and DICE and their basic use in the NDaST application.



Figure 2. Calculations of 1250 plutonium and mixed criticality benchmarks showing ENDF/B-VII.0 (red), CIELO-1 (blue) and cases where one component of the CIELO-1 evaluations were added to the ENDF/B-VII.1 evaluations: capture cross section (green), fission cross section (yellow) and average neutron multiplicity (purple). 


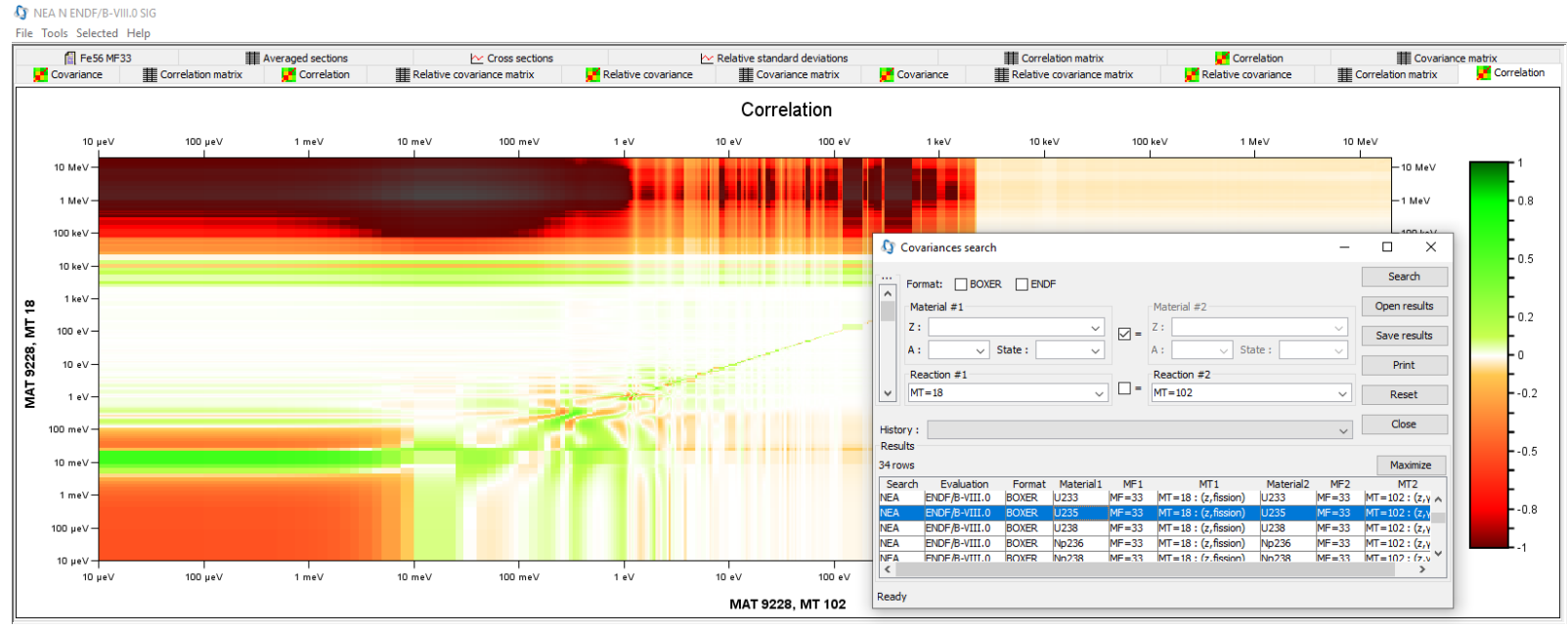

Figure 3. Cross-correlation covariance matrices from ENDF/B-VIII.0 between the fission and capture cross sections, shown using JANIS and reading from the NEA JANIS database.

Table 2. Summary of the new nuclear data libraries added to JANIS since the ND2016 conference.

\begin{tabular}{lc}
\hline Data Type & Included Datasets \\
\hline Radioactive data & ENDF/B-VIII.0, GEFY-6.2, \\
& JEFF-3.3, JENDL/DDF-2015 \\
\hline Incident neutron data & ENDF/B-VIII.0, GEFY-6.2, \\
& JEFF-3.3, JENDL-4.0/HE, \\
& JENDL/AD-2017, TENDL-2017 \\
\hline Incident gamma data & ENDF/B-VIII.0, JENDL/PD-2016, \\
& TENDL-2017 \\
\hline Incident proton data & ENDF/B-VIII.0, JENDL-4.0/HE, \\
& JENDL/AD-2017 \\
\hline Incident d,t,h,a data & ENDF/B-VIII.0, TENDL-2017 \\
\hline
\end{tabular}

who are preparing to add functionality in JANIS to accomodate new data. Complete processing of covariances for full energy-dependent emitted angle and energy-spectra data will be added alongside plans for extended sensitivity data. These may also be provided by the Validation of Nuclear Data Libraries (VaNDaL) Subgroup 45 and Subgroup 47, which are designing cross-comparison schema including sensitivity profiles and nominal calculation results.

As nuclear data library development projects design more automated systems for verification and validation, WPEC Subgroup 49 is working to design Continuous Integration pipelines for testing. These will benefit greatly from the rapid testing that NDaST offers. Just as with JANIS, the development of a command line interface (CLI) and/or an application programme interface (API) for NDaST is being investigated to directly integrate NDaST into automatic benchmarking workflows.

For more information, user manuals, direct launching and downloads, visit the NEA webpages: www.oecd-nea.org/janis www.oecd-nea.org/dice www.oecd-nea.org/ndast

\section{References}

[1] N. Soppera, M. Bossant, E. Dupont, Nuclear Data Sheets 120, 294 (2014)

[2] N. Otuka, E. Dupont, V. Semkova, B. Pritychenko, A. Blokhin, M. Aikawa, S. Babykina, M. Bossant, G. Chen, S. Dunaeva et al., Nuclear Data Sheets 120, 272 (2014)

[3] NEA, International Co-operation in Nuclear Data Evaluation: An Extended Summary of the Collaborative International Evaluated Library Organisation (CIELO) Pilot Project, NEA No. 7498, OECD, Paris (2019)

[4] M. Chadwick, R. Capote, A. Trkov, M. Herman, D. Brown, G. Hale, A. Kahler, P. Talou, A. Plompen, P. Schillebeeckx et al., Nuclear Data Sheets 148, 189 (2018), special Issue on Nuclear Reaction Data

[5] I. Hill, S. Jeong, Status and Analysis of P1 Angular Scattering Sensitivity Data Available Within the Database for ICSBEP (DICE), M\&C 2017, Jeju, Korea, April 16 - 20, 2017

[6] J. Dyrda, N. Soppera, I. Hill, M. Bossant, J. Gulliford, EPJ Web Conf. 146, 06026 (2017)

[7] J. Dyrda, I. Hill, L. Fiorito, O. Cabellos, N. Soppera, EPJ Nuclear Sci. Technol. 4, 14 (2018)

[8] A. Koning, D. Rochman, J.C. Sublet, N. Dzysiuk, M. Fleming, S. van der Marck, Nuclear Data Sheets 155, 1 (2019), special Issue on Nuclear Reaction Data

[9] L. Fiorito, G. Žerovnik, A. Stankovskiy, G. Van den Eynde, P. Labeau, Annals of Nuclear Energy 101, 359 (2017) 